\title{
ENTROPY MAXIMIZATION ALGORITHM FOR POSITRON EMISSION TOMOGRAPHY
}

\author{
Partha Pratim Mondal and K. Rajan* \\ Department of Physics, Indian Institute of Science \\ Bangalore 560012 , INDIA
}

\begin{abstract}
The expectation maximization (EM) algorithm is extensively used for tomographic image reconstruction based on Positron Emission Tomography (PET) modality. The EM algorithm gives good reconstructed images compared to those created by deterministic methods such as Filtered Back Projection (FBP) and Convolution Back projection (CBP). However, the computational complexity of EM-based algorithm is high due to the iterative nature of the algorithm. Prior knowledge of the estimate has been added to the basic EM algorithm to improve image quality as well as to reduce the number of iterations required for an acceptable image quality. We have developed an algorithm which produces better quality images in much lesser number of iterations, thereby speeding up the image reconstruction task.
\end{abstract}

Index Terms-Entropy Maximization, Poisson Process, Conditional Probability, Conditional Entropy

\section{INTRODUCTION}

The PET is an imaging technique, which is potentially useful in the study of human physiology and organ functions. The PET aims at obtaining a quantitative map of spatial and temporal distribution of the radio-nucleids inside the human body by measuring the event counts of positron-electron annihilation. The PET images can be reconstructed by either analytic methods such as CBP algorithm or by iterative algorithm such as EM algorithm. An analytic algorithm usually consists of two main computations. One is filtering and the other is backprojection. An iterative algorithm, on the other hand starts with an initial guess of the solution and iteratively updates the object according to computed pseudoprojections and measured projection data. But, the time for reconstruction of images using $\mathrm{EM}$ algorithm for obtaining an acceptable image is very high. A large number of algorithms has been proposed to accelerate the execution of $\mathrm{EM}$ algorithm as well as for improving the image quality. The EM algorithm and many variants of it attempt to maximize the likelihood function which is the probability

* School of Computer Engineering, Nanyang Technological University, Singapore 739 798. asrkrodan@ntu.edu.sg

This work was supported in part by Council of Scientific and Industrial Research CSIR, India. of observing the measured data given the emission parameter $\lambda$. We have addressed this problem in a much general form via entropy maximization (maximizing the information content) associated with the emission process rather than maximizing the likelihood function. This generalized form enables us to determine the form of prior knowledge about the object being imaged, which is not possible till now by any other means. Once prior knowledge about the object is known, then one can hope to have a reconstructed image of better quality.

Section II presents the entropy maximization and its relevance to PET. In section III, we bring out the form of the prior distribution. Section IV gives the implementation details. Section $\mathrm{V}$ discusses the results from the point of view of both quality of the image and the speed of reconstruction. Section VI concludes the paper.

\section{ENTROPY MAXIMIZATION}

The measurements in PET, $y_{j}, j=1 .,,, M$ are modeled as independent Poisson variables with mean parameters

$\sum_{j=1}^{M} \lambda_{i} p_{i j} \quad \mathrm{j}=1, \ldots, \mathrm{M}$

where

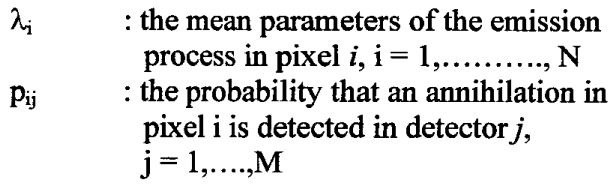

The conditional probability for observing $y_{j}$ given emission parameter $\lambda_{i}$ is given by Poisson distribution and can be expressed as,

$P(y / \lambda)=\prod_{j=1}^{M} \frac{e^{-\sum_{i=1}^{N} \lambda_{i} p_{i j}}\left(\sum \lambda_{i} p_{i j}\right)^{y_{j}}}{y_{j} !}$

The corresponding conditional entropy associated with the random process $y$ given emission parameter $\lambda$ is,

$H(y / \lambda)=\sum_{\lambda} P(\lambda) \cdot\left(P(y / \lambda) \cdot \log \left(\frac{1}{P(y / \lambda)}\right)\right.$ 
Substituting for $P(y / \lambda)$ from equation (1) into equation (2) and taking the first derivative of $H(y / \lambda)$, we get,

$$
\begin{aligned}
& \frac{\partial}{\partial \lambda_{i}}(H(y / \lambda))= \\
& -\sum_{i=1}^{N}\left\{\left(\frac{\partial}{\partial \lambda_{i}} P\left(\lambda_{i}\right)\right) \cdot\left(\prod_{j=1}^{M} \frac{e^{-\sum_{i^{\prime}=1}^{N} \lambda_{t} \cdot p_{i j}} \cdot\left(\sum_{i^{\prime}=1}^{N} \lambda_{i t^{\prime}} p_{i^{\prime} j}\right)^{y_{j}}}{y_{j} !}\right)\right. \\
& \left.\cdot\left(\sum_{j=1}^{M}\left(-\sum_{i^{\prime}=1}^{N} \lambda_{i^{\prime}} p_{i^{\prime} j}+y_{j} \cdot \log \left(\sum_{i^{\prime}=1}^{N} \lambda_{i^{\prime}} p_{i^{\prime} j}\right)-\log \left(y_{j}\right)\right)\right)\right) \\
& +P\left(\lambda_{i}\right)\left(\prod_{j=1}^{M} \frac{e^{-\sum_{i=1}^{N} \lambda_{i^{\prime}} p_{i j}} \cdot\left(\sum_{i^{\prime}=1}^{N} \lambda_{i^{\prime}} p_{i^{\prime} j}\right)^{y_{f}}}{y_{j} !}\right) \\
& \cdot\left(\sum_{j=1}^{M}\left(-\sum_{i^{\prime}=1}^{N} \lambda_{i^{\prime}} p_{i^{\prime} j}+y_{j} \cdot \log \left(\sum_{i^{\prime}=1}^{N} \lambda_{i^{\prime}} p_{i^{\prime}}\right)-\log \left(y_{j} !\right)+1\right)\right) \\
& \left.\left\{-\sum_{j=1}^{M} p i j+\sum_{j=1}^{M} \frac{y_{j .} p_{i j}}{\sum_{i^{\prime}=1}^{N} \lambda_{i^{\prime}} p_{i^{\prime}}}\right)\right\}
\end{aligned}
$$

Using Kuhn-Tuker conditions for an estimate $\hat{\lambda}$ to be a maximizer and the assumption $[\log (p(y / \lambda))+1 \approx$ $\log (p(y / \lambda))]$ we get,

$$
\begin{gathered}
\lambda_{i}^{k+1}=\frac{\lambda_{i}^{k}}{P\left(\lambda_{i}^{k}\right) \cdot \sum_{j=1}^{M} p_{i j}}\left(\frac{\partial P\left(\lambda_{i}^{k}\right)}{\partial \lambda_{i}}+P\left(\lambda_{i}^{k}\right) \cdot \sum_{j=1}^{M} \frac{y_{j} p_{i j}}{\sum_{i^{\prime}=1}^{N} \lambda_{i^{\prime}}^{k} p_{i^{\prime}}}\right) . \\
\quad \quad \text { where } \mathrm{i}=1, \ldots, \mathrm{N}
\end{gathered}
$$

Note that as the proposed recursion relation ( equation (4)) approaches EM-algorithm when $\mathrm{p}\left(\lambda_{\mathrm{i}}\right) \rightarrow$ uniform distribution.

$$
\hat{\lambda}_{i}^{\hat{k+1}}=\frac{\hat{\lambda}_{i}^{k}}{\sum_{j=1}^{M} p_{i j}} \sum_{j=1}^{M} \frac{y_{j} p f_{i j}}{\sum_{i^{\prime}=1}^{N} \hat{\lambda}_{i^{\prime}}^{k} p_{i j}}
$$

It can be seen that the proposed algorithm is much more general than EM-algorithm.

\section{DETERMINATION OF PRIOR DISTRIBUTION}

Maximum entropy algorithm enables us with the determination of prior distribution. Self-normalizing property of preserving the total activity of the estimate a constant gives,

$\sum \hat{\lambda}_{i}^{k+1}=c, \quad$ where $\mathrm{c}$ is a constant
Expanding,

$$
\sum_{i=1}^{N} \frac{\hat{\lambda}_{i}^{k}}{P\left(\hat{\lambda}_{i}^{k}\right) \cdot \sum_{j=1}^{M} p_{i j}}\left(\frac{\partial P\left(\hat{\lambda}_{i}^{k}\right)}{\partial \lambda_{i}}+P\left(\hat{\lambda}_{i}^{k}\right) \cdot \sum_{j=1}^{M} \frac{y_{j} p_{i j}}{\sum_{i^{\prime}=1}^{N} \hat{\lambda}_{i}^{k} p_{i j}}\right)=c
$$

Simplification gives,

$$
\sum_{i=1}^{N} \frac{\hat{\lambda}_{i}^{k}}{P\left(\hat{\lambda}_{i}^{k}\right)} \cdot \frac{\partial}{\partial \lambda_{i}^{k}} P\left(\hat{\lambda}_{i}^{k}\right)=c_{1}
$$

where

$$
c_{1}=c \sum_{j=1}^{M} p_{i j}-\sum_{j=1}^{M} y_{j} \text { is a constant. }
$$

Now, let us assume,

$$
\frac{1}{P\left(\hat{\lambda}_{i}^{k}\right)} \frac{\partial P\left(\hat{\lambda}_{i}^{k}\right)}{\partial \lambda_{i}}=a
$$

where $\mathbf{a}$ is a constant.

Therefore equation (5) becomes,

$$
\sum_{i=1}^{N} \hat{\lambda}_{i}^{k}=c
$$

Hence, self-normalization property is satisfied.

Solution to equation (7) is given by,

$$
P\left(\hat{\lambda}_{i}^{k}\right)=e^{a \hat{\lambda}_{i}^{k}}
$$

where $a$ is unknown.

Now, substituting equation (8) in equation (4) we get,

$$
\lambda_{i}^{k+1}=\frac{a \hat{\lambda}_{i}^{k}}{\sum_{j=1}^{M} p_{i j}}+\frac{\hat{\lambda}_{i}^{k}}{\sum_{j=1}^{M} p_{i j}} \sum_{j=1}^{M} \frac{y_{j} p_{i j}}{\sum_{i=1}^{N} \lambda_{i}^{k} p_{i j}}
$$

Equation (9) has not shown much improvement as the number of iteration increases. Rather, the following form of prior distribution $P(\lambda)$ has given tremendous improvement with a very small change in power of $\lambda$ i.e.,

$$
P\left(\hat{\lambda}_{i}^{k}\right)=\exp \left(a \cdot\left(\lambda_{i}\right)^{a}\right)
$$


Along with this form of prior and writing equation (9) in additive form gives,

$$
\hat{\lambda}_{i}^{k+1}=\hat{\lambda}_{i}^{k}+\frac{a \cdot\left(\lambda_{i}^{k}\right)^{a}}{\sum_{j=1}^{M} p_{i j}}+\frac{\left(\lambda_{i}^{k}\right)^{a}}{\sum_{j=1}^{M} p_{i j}} \cdot\left(\sum_{j=1}^{M} \frac{y_{j}-\sum_{i^{\prime}=1}^{N} \hat{\lambda}_{i}^{k} p_{i^{\prime} j}}{\sum_{i^{\prime}=1}^{N} \hat{\lambda}_{i}^{k} p_{i^{\prime} j}} \cdot p_{i j}\right)
$$

Equation (11) is the recursive relation. We call this maximum entropy (ME) algorithm.

\section{IMPLEMENTATION}

The proposed algorithm was implemented in a simulated PET system consisting of $64 \times 64$ pixels and a circular ring geometry of 64 detectors. The test phantom is shown in Figure 1. The pixel-detector probabilities were calculated as the angle of view of the $j^{\text {th }}$ detector tube from the center of the pixel $i$. Exploiting the eight fold symmetries in the system these probabilities were precomputed and stored as an array. Also, in the current study, the effects of factors such as photon attenuation and scattering are not taken into account. We are interested only in the evaluation of the proposed algorithm.

The reconstructed images using both EM and Entropy maximization algorithms are shown in Figure 2. The proposed algorithm gives better reconstructed images compared to those created by EM algorithm The number of iterations is considerably reduced.

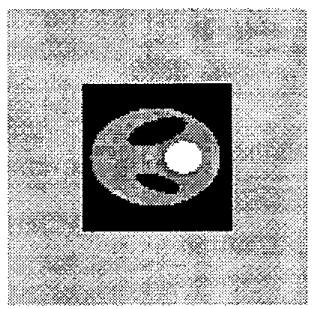

Figure 1 Original Test Phantom

\section{CONCLUSION}

The following are the conclusions on the basis of the proposed algorithm :

(1) The number of iterations has reduced considerably.

(2) Image quality is found to be much superior than the image obtained by EM-algorithm.

Moreover, one can notice that as the parameter $a$ varies from 1.0 to 1.063 , the number of iterations for getting better images also reduces.

\section{ACKNOWLEDGEMENT}

The first author express his thanks to Hemanth T., research scholar, Department of Physics, Indian Institute of Science for his support and help during programming. Also, he wishes to thank CSIR for financial support to undertake the work.

\section{REFERENCES}

[1] L.A. Sheep and Y. Vardi, "Maximum likelihood estimation for emission tomography," IEEE Trans. on Medical Imaging, MI-1: pp. 113-121, 1982

[2] T. Hebert and R. Leahy, "A generalized EM algorithm for 3-D Bayesian reconstruction from Poisson data using Gibbs priors," IEEE Trans. on Medical Imaging, MI-8(2) : pp. 194-202, 1989.

[3] E. Levitan and G.T. Herman, "A maximum a posteriori probability expectation maximization algorithm for image reconstruction in emission tomography," IEEE Trans. on Medical Imaging, MI-6(3) : pp. 185-192, 1987.

[4] K. Lange, M. Bahn, and R. Little, "A theoretical Study of some maximum likelihood algorithm for emission and transmission tomography," IEEE Trans. on Medical Imaging, MI-6(2) : pp. 106-114, 1987

[5]N. Rajeevan "Stochastic estimation approach to emission tomography," Ph D Thesis, Department of Electrical Engineering, Indian Institute of Science, November 1991, Bangalore India.

[6] A. Dempster, N. M. Laird, and D. B. Rubin, " Maximum Likelihood from incomplete data via the EM algorithm, "J. Roy. Statist. Soc., ser. B, vol. 39, pp. 1-38, 1977.

[7] K. Lange and R. Carson, "EM reconstruction algorithms for emission and transmission tomography, $J$. Comput. Assist. Tomog. , vol. 8, pp. 306-316, 1984.

[8] N. Rajeevan, K. Rajgopal, and G. Krishna, "Vector extrapolated fast maximum likelihood estimation algorithms for emission tomography," IEEE Transactions on Medical Imaging, vol.11, No.1, March 1992.

[9] Avinash C. Kak and Malcolm Slaney, "Principles of computerized Tomographic Imaging, "IEEE Press, 1988.

[10] Gabor T. Herman, "Image Reconstruction from Projection," Academic Press, 1980 .K.

[11] K. Lange, M. Bahn, and R. Little, "A theoretical study of some maximum likelihood algorithm for emission and transmission tomography," IEEE Trans. on Medical Imaging, vol. MI-6, pp. 106-114, June, 1987. 


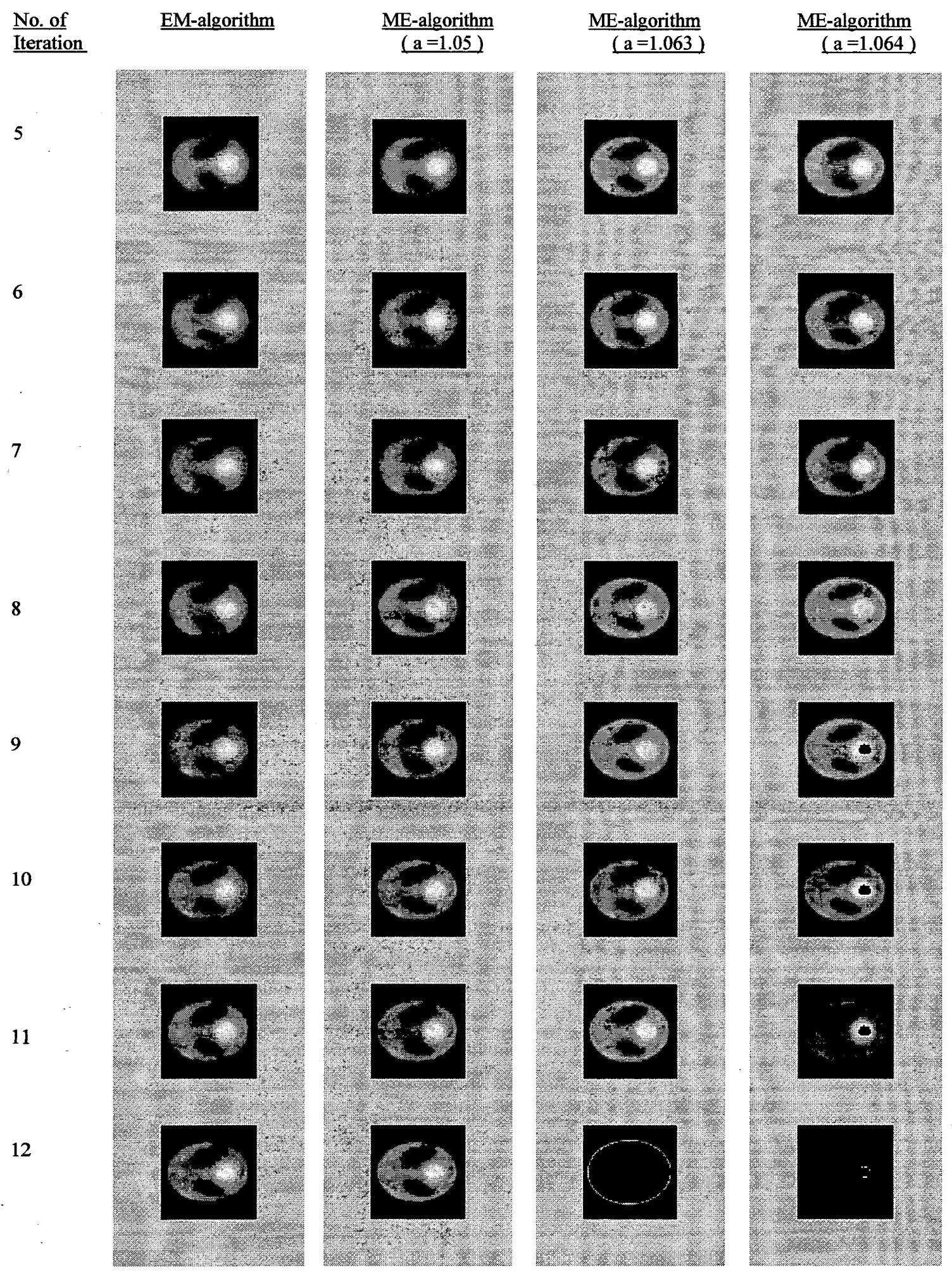

Figure 2 The reconstructed images using EM algorithm and the proposed Entropy Maximization Algorithms 